\title{
REPTILES DOMESTIQUES (NAC) ET SALMONELLA
}

\author{
DOMESTIC REPTILES AND SALMONELLA
}

Par Alain PHILIPPON ${ }^{1}$

(Lettre envoyée le 20 mars 2021, manuscrit accepté le 22 mars 2021)

Mots-clés : Salmonella, habitat, identification, sérotype, épidémiologie, reptile, tortue, iguane, serpent, lézard, NAC.

Key words: Salmonella, habitat, identification, serotype, epidemiology, reptile, turtle, iguana, snake, lizard.

\section{INTRODUCTION}

Une intéressante et récente séance de l'AVF sur les Actualités en herpétologie a soulevé plusieurs interventions sur le rôle des reptiles, Nouveaux Animaux de Compagnie (NAC), dans la transmission des Salmonella qui a été particulièrement démontrée lors d'infections infantiles dès les années 1960 (Colomb-Cotinat et al. 2014a ; Colomb-Cotinat et al. 2014b) ; cette étude a ensuite été fort bien relayée sur le Web. Parmi ces reptiles, il convient de citer, par ordre de prévalence, les tortues, les lézards, les iguanes, et certains serpents non venimeux dont la vente est réglementée dans certains pays tels les USA pour les petites tortues dès 1975, ou encore le Canada. L'implication des Salmonella est bien connue dans les Toxi-Infections Alimentaires Collectives (TIAC), en particulier chez l'adulte ; une surveillance sanitaire lors d'épidémie impose des investigations très poussées, en particulier au plan bactériologique, compte tenu de l'extrême complexité de ce genre bactérien.

\section{TAXONOMIE}

Les Salmonella appartiennent à la famille des entérobactéries (bacilles à Gram négatif aéro-anaérobies fermentaires) ; la complexité de leur taxonomie s'est accrue avec leur individualisation en deux espèces: Salmonella enterica, majoritaire, et Salmonella bongori. L'espèce S. enterica est identifiable en sous-espèces : azizonae, diarizonae, enterica, houtenae, indica et salamae (Tindall et al. 2005). L'espèce Salmonella subterranea a été finalement reclassée dans le nouveau genre Atlantibacter avec l'ancienne espèce Escherichia hermannii (Hata et al. 2016). Malgré la complexité antigénique des Salmonella, les antigènes $\mathrm{O}$ de leur paroi et $\mathrm{H}$ de leurs flagelles permettent une identification en sérotypes ou sérovars, le séquençage du génome complet (WGS pour Whole Genome Sequencing) autorise maintenant une approche beaucoup plus précise et plus de 110.000 exemplaires sont accessibles sur EnteroBase (Alikhan et al. 2018) disponible à https://enterobase.wa rwick.ac.uk. Ainsi la sous-espèce très majoritaire, S. enterica subsp. enterica comprend plus de 2500 sérotypes. Il est évident que leur identification ne peut être menée que par un laboratoire spécialisé, en l'occurrence le Centre National de Référence des Escherichia coli, Shigella, Salmonella (CNR-ESS) (www.pasteur.fr/fr/sante-publique/CNR/les-cnr/escher ichia-coli-shigella-salmonella). Une telle complexité permet d'effectuer des enquêtes épidémiologiques très précises ou encore d'établir une surveillance épidémiologique (voir fiche de renseignements épidémiologique CNR-ESS, figure 1). Ainsi nous disposons au plan national et international de données précises sur cette zoonose dont le nombre d'épidémies en France a quadruplé depuis 2018 (figure 2). Certains sérotypes ont une prévalence importante lors de TIAC au plan mondial, tels S. enterica ser. Typhimurium, S. enterica ser. Enteritidis d'autres, tels S. enterica subsp. azizonae, S. enterica subsp. diarizonae, S. enterica subsp. houtenae, S. enterica subsp. indica et S. enterica subsp. salamae sont préférentiellement observés chez les reptiles (Colomb-Cotinat et al. 2014a ; 2014b; Pulford et al. 2019).

(1) Professeur Émérite, Faculté de Médecine, Université de Paris. Courriel : fephillips66@gmail.com 

Institut Pasteur
Centre National de Référence des Escherichia coli, Shigella et Salmonella Unité des Bactéries Pathogènes Entériques Institut Pasteur

28, rue du Docteur Roux - 75724 PARIS CEDEX 15

TEL : $01456883 \quad 39$-FAX: 0145688837 -e-mail : salmonella@pasteur.fr Dr Maria PARDOS DE LA GANDARA - Dr Sophie LEFEVRE - Dr François-Xavier WEILL

Fiche de renseignement devant accompagner chaque envoi (téléchargeable à partir de notre site internet) : https://mww.pasteur.fr/sites/default/files/rubrique pro sante publique/les cnr/escherichia coli shigella salmonella/ficheinfosalmonella dec2020.pdr

\section{SALMONELLA}

Les champs grisés sont INDISPENSABLES.

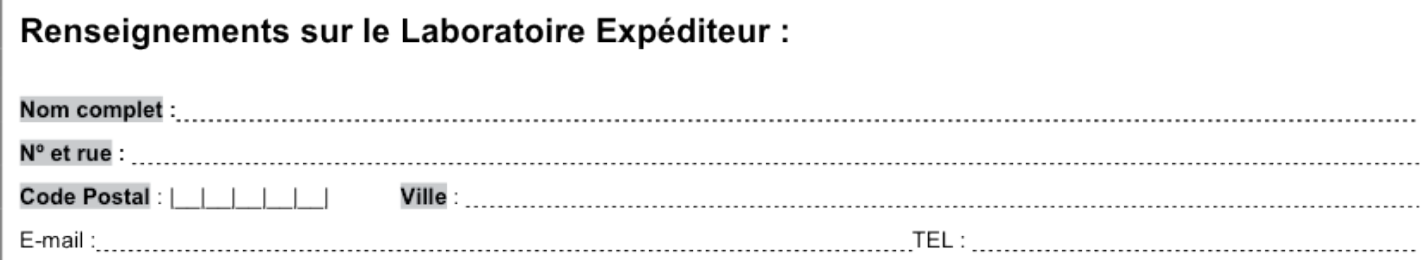

\section{Renseignements sur le Patient :}

Nom, Prénom / Référence

Sexe : F / M

Date de naissance : $\left|\_\right| /\left|\_\right| / \mid$

Code Postal (domicile) : L__ _ _ _ _ _ _ l

Statut : $\quad$ malade $L$ _ $\quad$ porteur $\mathrm{L} \_$linconnu $L$

Cas :

Isolé L_ | Groupés L_ |

Nombre total de cas

Familiaux $\downarrow$ Hospitaliers $\downarrow$

Scolaires $\mid+\quad$ Crèche $|+|$

Professionnels $\perp \mid$ Autres $\perp$

Toxi-infection alimentaire collective (TIAC) L

Aliment suspecté

Voyage récent (pays, date)

Présence de nouveaux animaux de compagnie au domicile (N.A.C.) : L

Reptiles :

\section{Renseignements sur l'échantillon :}

Origine de la souche : Sang $\mid \_$Selles $\mid \_$Urines $\mid$ Date de prélèvement : $\left|\_\right| /\left|\_\right| /\left|\_\right| \_\left|\_\right|$

Identification trouvée :

Sous-espèce

Sérotype
Prélèvement NON humain :

(Analyse facturable, réalisé après entente préalable avec les responsables du CNR)

\section{Référence :}

Nature :

Vétérinaire $\mathrm{L}$

Alimentaire |

Environnemental $\_$।

\section{Précisez}

\section{Date de prélèvement}

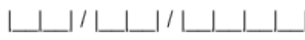

Origine géographique :

Informations complémentaires :

\section{Merci de joindre une copie de l'antibiogramme réalisé}

Pour toutes demandes ou renseignements particuliers, veuillez nous contacter préalablement par téléphone (014568 0339 ) ou par courriel ( $\underline{\text { salmonella@pasteur.fr) }}$

Nous vous remercions pour votre collaboration à la surveillance épidémiologique des infections dues aux Salmonella.

Le CNR étant informatisé et n'ayant pas de contact direct avec les patients, nous vous remercions d'informer ceux-ci de leur droit d'accès et de rectification des informations les concernant (Loi Nº78-17 du 06 janvier 1978). Version 04/12/2020

Figure 1: Fiche de renseignement épidémiologique (CNR ESS, Institut Pasteur Paris) 
Nombre

d'épidémies

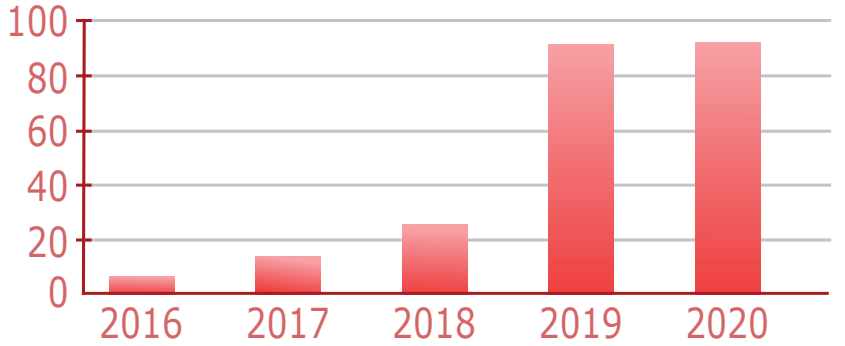

Figure 2 : Nombre d'épidémies détectées en France par le CNR-ESS L'utilisation du WGS en première intention est effective au CNR-ESS depuis juillet 2018 \# Données partielles pour 2020 (au 20/11/2020), la surveillance épidémiologique ayant été fortement affectée par la pandémie COVID-19. (https://www.pasteur.fr/fr Lfile/37997/download)

\section{HABITAT}

Ces bactéries "telluriques " peuvent survivre plusieurs mois dans l'eau, aussi la contamination fécale suite à un portage digestif animal (bovins, ovins, volailles), voire humain lors d'une épidémie de fièvre typhoïde ou paratyphoïde, est-elle possible. Elles peuvent aussi contaminer des aliments tels viande, lait ou ouf, et déclencher chez le consommateur une TIAC. Il est essentiel de savoir que certaines espèces ou sous-espèces de Salmonella sont naturellement hébergées par le tube digestif des reptiles (animaux à sang froid). Cependant, la cavité buccale apparait peu colonisée si l'on se réfère à la très faible prévalence de salmonelloses humaines après morsure par des serpents (August et al. 2018). En revanche, plusieurs études dans le monde rapportent le rôle prépondérant des petites tortues porteuses de S. enterica subsp. arizonae amenant à exclure leur commercialisation aux USA dès 1975.

\section{CLINIQUE}

Classiquement, en dehors des fièvres septicémiques (fièvre typhoïde par exemple), l'infection par Salmonella est à dominante digestive (vomissement, diarrhée). Chez l'enfant, l'analyse bibliographique parue dans le BEH et portant sur 43 publications dans divers pays (Europe (23), USA (16) ou encore Japon, Inde, Australie) rapporte 77 infections, dont 49 chez les moins d'un an (Colomb-Cotinat et al. 2014b). Chez l'enfant, mais aussi l'adulte immunodéprimé ou âgé, il s'agit majoritairement, dans les deux tiers des infections, de cas isolés, de gastro-entérites rarement mortelles ; un tiers se manifeste par des septicémies, arthrites, ostéomyélites ou encore de méningites (11 cas) chez l'enfant de moins de six mois. Si un seul de ces onze enfants est décédé, deux ont présenté des retards de développement et les suites ont été favorables pour les huit autres. Deux cas de co-infection avec le Rotavirus ont été rapportés en France (Angot et al. 2017).

\section{ÉPIDÉMIOLOGIE}

Le diagnostic bactériologique permet de préciser que les souches de Salmonella isolées appartiennent majoritairement à la sous-espèce enterica et les reptiles les plus fréquemment impliqués sont les tortues et les serpents (Colomb-Cotinat et al. 2014a ; 2014b). Par ailleurs, de l'ordre de 50 à 90\% des reptiles sont porteurs sains dans leur tube digestif. La survie de ces bactéries dans l'environnement après défécation est possible pendant plusieurs semaines en raison des conditions de température et d'humidité favorables, justifiant des mesures d'hygiène particulières. Si une grande diversité de sérotypes est habituellement rapportée dont ceux habituellement isolés lors de TIAC chez l'adulte, la revue bibliographique sur les formes infantiles rapporte que 60 infections sur 77 sont dues à S. enterica ser. Enterica, Les autres sérotypes appartiennent dans presque un quart des cas aux sous-espèces houtenae (8 infections), arizonae (7 infections) ou encore diarizonae ( 2 infections), ces dernières espèces étant habituellement hébergées par les reptiles (Colomb-Cotinat et al. 2014a ; 2014b). La contamination s'effectue le plus souvent par contact direct avec le reptile d'autant que le jeune enfant aura tendance à prendre l'animal et le porter à la bouche. Cependant, la contamination indirecte est aussi décrite lors du nettoyage de la cage dans la cuisine à proximité de la vaisselle familiale. Un autre exemple de contamination indirecte, très inhabituel, concerne un usage folklorique, propre aux hispaniques comme au Mexique, à savoir l'ingestion de pilules, de capsules, voire de poudres contenant des extraits de crotale parées de diverses vertus médicinales, malheureusement ingérées par des malades immunodéprimés (cancer, VIH...) (CDC 1983, Martínez-Barreda et al. 1991 ; Noskin \& Clarke 1990). Il s'agissait le plus souvent de S. enterica subsp. arizonae. Enfin, des épidémies ont été rapportées dans plusieurs pays (Canada, Royaume-Uni, USA) en relation avec la nourriture contaminée (souris congelée) par des Salmonella donnée aux serpents (Fuller et al. 2008 ; Marin et al. 2018 ; Vrbova et al. 2018). Par exemple, l'épidémie canadienne a perduré trois ans, étendue à cinq provinces et totalisant 134 cas dont $45 \%$ d'enfants de moins de 12 ans (Vrbova et al. 2018).

\section{MESURES PRÉVENTIVES}

Les salmonelloses restent la première zoonose mondiale habituellement caractérisée par un syndrome digestif chez l'adulte (gastro-entérite) rarement mortel. Cependant, le contexte épidémiologique particulier, propriétaire possédant un reptile dans la famille avec au moins un enfant, voire un parent immunodéprimé, amène à être précautionneux. Peu de propriétaires sont conscients du danger de leur NAC. Aussi diverses mesures épidémiologiques concernant la conservation des reptiles NAC, leur habitat, les consignes de manipulation ont-elles été prises par les autorités sanitaires des pays concernés. Le CNR-ESS rapporte aussi qu'en France, des souches de $S$. Typhimurium résistantes aux fluoroquinolones (FQ) ont été isolées dès 2008 chez des serpents. Compte-tenu du portage sain 
des Salmonella par ces reptiles, l'enrofloxacine (FQ) a certainement été utilisée préventivement dans des animaleries (cf. rapport d'activité CNR-ESS 2008 disponible à https://www.pasteur.fr/fr $\angle$ sante-publique/CNR/les-cnr/escherichia-coli-shigella-salm onella/rapports-d-activite).

Cette résistance acquise est d'autant plus préjudiciable que le mécanisme de résistance est identique à celui observé dans des souches d'origine humaine : mutations ponctuelles dans les gènes de gyrases : gyrA et gyrB.

Si les responsables d'animalerie connaissent la réglementation sur les reptiles très variable selon les pays, le particulier le plus souvent ignorant de ce risque de contamination devra toujours respecter un certain nombre de recommandations : ne pas garder de reptiles s'il y a un enfant dans le foyer, se laver les mains à l'eau savonneuse lors de la préparation du repas familial, baigner le reptile dans un bassin exclusivement réservé à cet usage et non dans sa salle de bain, rejeter l'eau dans les toilettes. Toute surface avec laquelle un reptile est entré en contact doit être considérée comme contaminée, la laver à l'eau savonneuse ou avec de l'eau de javel, de même se laver les mains après les soins au reptile et la manipulation de sa nourriture. Ne pas porter les mains à la bouche après manipulation d'un reptile, éviter de manger ou de boire près d'eux ...Le Ministère de la santé de l'Ontario (Canada) diffuse des recommandations très précises à l'usage du grand public disponible sur le web à https://www.health.gov.on.ca/fr/public/publications/disease/sa lmonella arfr.aspx)

\section{CONFLIT D'INTÉRÊT}

L'auteur ne déclare aucun conflit d'intérêt dans la rédaction de cette note qui exprime son opinion personnelle.

\section{REMERCIEMENTS}

Merci Jean-Paul Rousseau si efficace dans la lecture de ce manuscrit.

\section{RÉFÉRENCES}

- Alikhan NF, Zhou Z, Sergeant MJ, Achtman M. A genomic overview of the population structure of Salmonella. PLoS Genet. 2018; 14:e1007261.

- Angot M, Labbé F, Duquenoy A, Le Roux P. Co-infection rotavirus-Salmonella lié aux tortues : à propos de deux cas de zoonoses domestiques. Arch Pédiatr. 2017;24:747-748.

- August JA, Boesen KJ, Hurst NB, Shirazi FM, Klotz SA. Prophylactic antibiotics are not needed following rattlesnake bites. Am J Med. 2018;131:1367-1371.

- Centers for Disease Control (CDC). Arizona hinshawii septicemia associated with rattlesnake powder-California. MMWR Morb Mortal Wkly Rep. 1983; 32:464-465.

- Colomb-Cotinat M, Le Hello S, Rosières X, Lailler R, Weill Fx, Jourdan-Da Silva N. Salmonelloses chez des jeunes enfants et exposition aux reptiles domestiques : investigation en France métropolitaine en 2012. Bull Épidémiol Hebd. 2014a; 1-2:2-8.

- Colomb-Cotinat M, Le Hello S, Rosières
X, Lailler R, Weill Fx, Jourdan-Da Silva $\mathrm{N}$. Cas publiés de salmonelloses chez les jeunes enfants secondaires à une exposition aux reptiles : revue bibliographique 1993-2013. Bull Épidémiol Hebd. 2014b; 1-2:8-16.

- Fuller CC, Jawahir SL, Leano FT, Bidol SA, Signs K, Davis C et al. A multi-state Salmonella Typhimurium outbreak associated with frozen vacuum-packed rodents used to feed snakes. Zoonoses Public Health. 2008;55:481-487.

- Hata H, Natori T, Mizuno T, Kanazawa I, Eldesouky I, Hayashi M et al. Phylogenetics of family Enterobacteriaceae and proposal to reclassify Escherichia hermannii and Salmonella subterranea as Atlantibacter hermannii and Atlantibacter subterranea gen. nov., comb. nov. Microbiol Immunol. 2016;60:303-311.

- Marin C, Martelli F, Rabie A, Davies R. Commercial frozen mice used by owners to feed reptiles are highly externally contaminated with Salmonella Enteritidis PT8. Vector Borne Zoonotic Dis. 2018;18:453-457.
- Márquez-Dávila G, Martínez-Barreda C, Suárez-Ramírez I. Cápsulas de víbora desecada: una fuente potencial de infección por bacterias gram negativas. Rev Invest Clin. 1991;43:315-317.

- Noskin GA \& Clarke JT. Salmonella arizonae bacteremia as the presenting manifestation of human immunodeficiency virus infection following rattlesnake meat ingestion. Rev Infect Dis. 1990; 12:514-517.

- Pulford CV, Wenner N, Redway ML, Rodwell EV, Webster HJ, Escudero R et al. The diversity, evolution and ecology of Salmonella in venomous snakes. PLoS Negl Trop Dis. 2019;13:e0007169.

Tindall BJ, Grimont PAD, Garrity GM, - Euzéby JP. Nomenclature and taxonomy of the genus Salmonella. Int J Syst Evol Microbiol. 2005;55:521-524.

Vrbova L, Sivanantharajah S, Walton - R, Whitfield Y, Lee C, Picard I, et al. Outbreak of Salmonella Typhimurium associated with feeder rodents. Zoonoses Public Health. 2018;65:386-394. 